\title{
P-Chirogenic Monodentate Phosphine Ligands Bearing 2-Biphenyl Group
}

\author{
Hideyuki Tsuruta, Tsuneo Imamoto* \\ Department of Chemistry, Faculty of Science, Chiba University, Chiba 263-0041, Japan \\ Fax +81-43-290-2791; E-mail: imamoto@ scichem.s.chiba-u.ac.jp \\ Received 1 February 2001 \\ This paper is dedicated to Professor Ryoji Noyori for his distinguished achievements in chemistry.
}

\begin{abstract}
Optically pure P-chirogenic monodentate phosphine ligands, [(S)-(2-biphenyl)(tert-butyl)methylphosphine $\quad((S)$-3a $]$, $(R)$-(2-biphenyl)cyclohexylmethylphosphine $[(R)-3 \mathbf{b}]$, and $(S)-(2-$ biphenyl)methylphenylphosphine $[(S)-3 \mathbf{c}]$ were synthesized via phosphine-borane intermediates. Palladium-catalyzed asymmetric allylic alkylation using $(S)$-3a and $(R)$-3b as the chiral ligands afforded products with the same absolute configuration in high enantioselectivity.
\end{abstract}

Key words: P-chirogenic phosphines, monodentate phosphine ligands, asymmetric allylic alkylation, asymmetric synthesis

Catalytic asymmetric synthesis using transition metal complexes with chiral ligands is one of the most powerful and economically promising methods for the synthesis of enantiomerically enriched compounds. ${ }^{1}$ In particular, chiral bidentate phosphine ligands have been most frequently used in asymmetric catalyses. On the other hand, chiral monodentate phosphine ligands also play a significant role in some asymmetric reactions. ${ }^{2,3}$ Moreover, it has been reported that sterically congested phosphines remarkably increase the catalytic activities of the metal complexes. ${ }^{4}$ These facts led us to develop new chiral monodentate phosphine ligands that are anticipated to exhibit high catalytic efficiency.

Previously, we reported that enantiomerically pure phosphines possessing asymmetric phosphorus atoms are synthesized by the use of phosphine-boranes as the intermediates. ${ }^{5}$ Based on this methodology, we tried to prepare enantiomerically pure alkyl(2-biphenyl)methylphosphines (alkyl = tert-butyl, cyclohexyl, and phenyl), because monodentate phosphines possessing 2-biphenyl group have been employed as effective ligands in asymmetric catalyses such as Pd-catalyzed carbon-carbon bond forming reactions. ${ }^{4 \mathrm{~b}-\mathrm{e}}$ tert-Butyldichlorophosphine, dichlorocyclohexylphosphine, and dichlorophenylphosphine were reacted successively with 2-lithiobiphenyl, lithium $l$-menthoxide, and borane-THF to give the corresponding diastereomer mixtures (ca. 1: 1) of key intermediates bearing 2-biphenyl and $l$-menthyloxy groups in $62-74 \%$ yields (Scheme 1). Each mixture was separated by preparative HPLC or recrystallization from hexane to give pure diastereomers. ${ }^{6}$ The absolute configurations around the chirogenic phosphorus atom of compounds $\left(R_{\mathrm{P}}\right)$-1b and $\left(R_{\mathrm{P}}\right)$-1c were unequivocally determined by single crystal X-ray analyses.

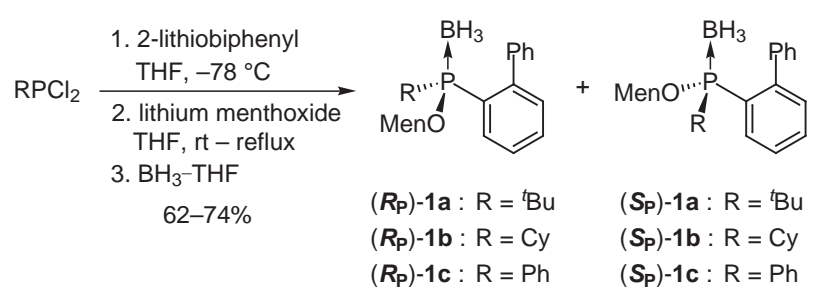

Scheme 1

Compound $\left(S_{\mathrm{P}}\right)$-1a was reduced by $4,4^{\prime}$ '-di-tert-butylbiphenylide (LDBB) at $-60{ }^{\circ} \mathrm{C}$, followed by the treatment with iodomethane, to give $(S)$-2a with $75 \%$ ee in $72 \%$ yield. ${ }^{7,8}$ Recrystallization of this product from hexane twice afforded enantiomerically pure compound, which in turn was dissolved in $\mathrm{N}$-methylpyrrolidine to remove the boranato group. The deboranation reaction was monitored by silica gel TLC, and after disappearance of the phosphine-borane the solvent was evaporated. The residual oil was purified by passing through a short column of silica gel using degassed toluene to afford [ $(S)$-(2-biphenyl)(tert-butyl)methylphosphine $(S)$-3a] in almost quantitative yield (Scheme 2).
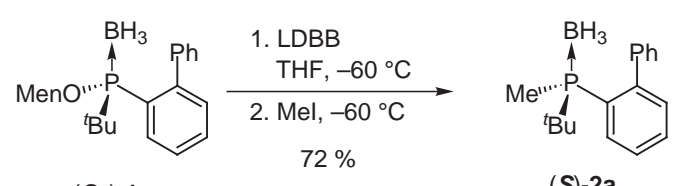

(Sp)-1a

(S)-2a

$75 \%$ ee (> 99\% ee after recry.)

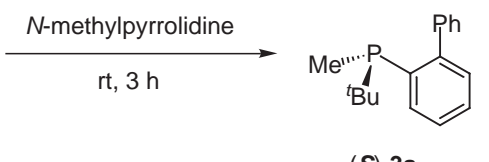

Scheme 2

The above mentioned procedure was not applicable for the preparation of optically active (2-biphenyl)cyclohexylmethylphosphine, because the reduction of $\left(R_{\mathrm{P}}\right)-\mathbf{1 b}$ by LDBB and subsequent methylation resulted in disappointedly low enantiomeric excesses (ca. 20\% ee) of the product, probably owing to rapid stereomutation of the generated tricoordinate phosphorus species. Therefore, 
we employed another promising method, although it required two more reaction steps. Thus, compound $\left(R_{\mathrm{P}}\right)-\mathbf{1 b}$ was subjected to deboranation by treatment with $N$-methylpyrrolidine at $50{ }^{\circ} \mathrm{C}$ and the generated phosphine was methylated with methyl triflate. The resulting phosphonium salt was stereospecifically reduced by $\mathrm{LiAlH}_{4} \cdot{ }^{9} \mathrm{Be}-$ cause of its air sensitivity, the product was converted to air stable borane adduct $(R)-\mathbf{2 b}(97 \%$ ee) in $73 \%$ overall yield from $\left(R_{\mathrm{P}}\right)-\mathbf{1 b}$. Enantiomerically pure $(R)-\mathbf{2 b}$, which was obtained by recrystallization from hexane, was transformed to desired monophosphine $(R)-3 \mathbf{b}$ by the reaction with $N$-methylpyrrolidine (Scheme 3 ).

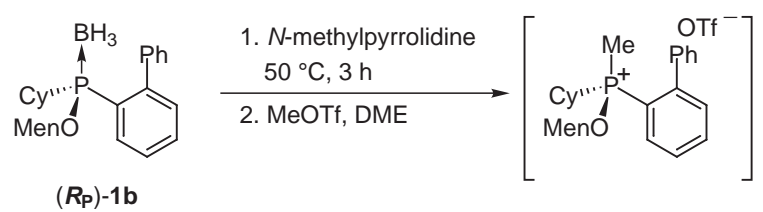

$$
\begin{gathered}
\text { 1. } \mathrm{LiAlH}_{4} \\
\text { 2. } \mathrm{BH}_{3}-\mathrm{THF}
\end{gathered}
$$

\section{Scheme 3}

The third ligand $(S)$-3c was readily prepared from $\left(R_{\mathrm{P}}\right)$-1c by nucleophilic substitution with methyllithium, followed by usual deboranation reaction (Scheme 4). It is noted that the substitution reaction proceeded in THF at $40{ }^{\circ} \mathrm{C}$ with inversion of configuration at phosphorus in $94 \%$ stereospecificity. ${ }^{10}$

$$
\text { MenO }
$$

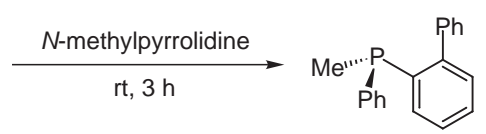

(S)-3c

Scheme 4

With these ligands in hand, we evaluated their enantioselectivity in palladium-catalyzed asymmetric allylic alkylation, a typical catalytic carbon-carbon bond forming reaction. ${ }^{11,12}$ The reactions were carried out using 1,3diphenyl-2-propenyl acetate (4) as a model substrate under various conditions. At first we tried the reaction of $\mathbf{4}$ with dimethyl malonate using $(S)-\mathbf{3 a}$ as the ligand in the presence of additives. The reactions proceed in $\mathrm{CH}_{2} \mathrm{Cl}_{2}$ at room temperature to give the coupling product in almost quantitative yield. No distinct differences in the enantioselectivity were observed by changing the additive (Entries 1-5 in Table), and therefore potassium acetate was used as the additive in other reactions. The reaction in $\mathrm{CH}_{2} \mathrm{Cl}_{2}$ gave the highest ee of the product among the solvents examined, and hence we used $\mathrm{CH}_{2} \mathrm{Cl}_{2}$ in this series of reactions.

It is noted that ligand $(R)-\mathbf{3 b}$ gave distinctly higher enantioselectivity than $(S)$-3a and in sharp contrast $(S)$-3c proved to be ineffective in this asymmetric reaction (Entries 10-12). Entries 13-19 show the results of the reactions with other active methylene compounds using $(S)$-3a and $(R)-\mathbf{3 b}$ as the ligands. $(R)-\mathbf{3 b}$ again afforded better enantioselectivity than $(S)-3 \mathbf{a}$. Another notable fact is that both $(S)-3 \mathbf{a}$ and $(R)-3 \mathbf{b}$ provided the coupling products with the same absolute configuration. These results can be interpreted by assuming that cyclohexyl group is sterically smaller than 2-biphenyl group and tert-butyl group is larger than 2-biphenyl group.

In summary, we have synthesized new enantiomerically pure P-chirogenic monodentate phosphine ligands possessing bulky groups, and demonstrated their utility in palladium-catalyzed asymmetric allylic alkylation.

\section{Acknowledgement}

We thank Dr. Karen V. L. Crépy for the preparation of compound 1b. This work was supported by the Research for the Future Program, the Japan Society for the Promotion of Science, the Ministry of Education, Culture, Sports, Science and Technology.

\section{References and Notes}

(1) (a) Noyori, R.; Asymnmmertic Catalysis in Organic Synthesis; John Wiley \& Sons: New York 1994. (b) Ojima, I.; Catalytic Asymmetric Synthesis, second edition; Wiley-VCH: New York 2000.

(2) For reviews dealing with monodenate phosphines, (a) Hayashi, T. Acc. Chem. Res. 2000, 33, 354. (b) Lagasse, F.; Kagan, H. B. Chem. Pharm. Bull. 2000, $48,315$.

(3) For recent examples of chiral monodentate phosphine ligands, (a) Hamada, Y.; Sato, N.; Ohmori, H.; Hatano, K. Tetrahedron Lett. 1996, 37, 7565. (b) Zhu, G.; Chen, Z.; Jiang, Q.; Xiao, D.; Cao, P.; Zhang, X. J. Am. Chem. Soc. 1997, 119, 3836. (c) Yin, J.; Buchwald, S. L. J. Am. Chem. Soc. 2000, 122, 12051. (d) van den Berg, M.; Minnaard, A. J.; Schudde, E. P.; van Esch, J.; de Veries, A. H. M.; de Vries, J. G.;

Feringa, B. L. J. Am. Chem. Soc. 2000, 122, 11539. (e) Reetz, M. T.; Mehler, G. Angew. Chem. Int. Ed. 2000, 39, 3889. (f) Claver, C.; Fernandez, E.; Gillon, A.; Heslop, K.; Hyett, D. J.; Martorell, A.; Orpen, A. G.; Pringle, P. G. Chem. Commun. 2000, 961. (g) Cammidge, A. N.; Crépy, K. V. L. Chem. Commun. 2000, 1723.

(4) For recent examples of catalytic reactions using sterically congested monodentate phosphines, (a) Mann, G.; Incarvito, C.; Rheingold, A. L.; Harrtwig, J. F. J. Am. Chem. Soc. 1999, 121, 3224. (b) Wolfe, J. P.; Simger, R. A.; Yang, B. H.; Buchwald, S. L. J. Am. Chem. Soc. 1999, 121, 9550. (c) Littke, A. F.; Dai, C.; Fu, G. C. J. Am. Chem. Soc. 2000, 122, 4020. 
Table Asymmetric Allylic Alkylation Catalyzed by Palladium Complexes Coordinated with Monodentate Phosphine Ligands.

\begin{tabular}{|c|c|c|c|c|c|c|c|c|}
\hline \multirow[b]{2}{*}{ entry } & \multirow[b]{2}{*}{ ligand } & \multirow{2}{*}{$\mathrm{Nu}$} & \multicolumn{2}{|c|}{$\begin{array}{l}1.5 \mathrm{~mol} \%\left[\mathrm{PdCl}\left(\mathrm{C}_{3} \mathrm{H}_{7}\right)\right]_{2}, \\
6 \mathrm{~mol} \% \text { ligand } \\
\text { 2eq. Nu-H, 2eq. BSA } \\
10 \mathrm{~mol} \% \text { additive }\end{array}$} & \multirow[b]{2}{*}{ temp } & \multirow{2}{*}{ time (h) } & \multirow{2}{*}{ yield $(\%)^{a}$} & \multirow[b]{2}{*}{ ee $(\%)$} \\
\hline & & & addtive & solvent & & & & \\
\hline 1 & $(S)-\mathbf{3 a}$ & $\mathrm{CH}(\mathrm{COOMe})_{2}$ & $\mathrm{KOAC}$ & $\mathrm{CH}_{2} \mathrm{Cl}_{2}$ & rt & 15 & $>99$ & 80 \\
\hline 2 & $(S)-\mathbf{3 a}$ & $\mathrm{CH}(\mathrm{COOMe})_{2}$ & LiOAc & $\mathrm{CH}_{2} \mathrm{Cl}_{2}$ & $\mathrm{rt}$ & 17 & $>99$ & 65 \\
\hline 3 & $(S)-\mathbf{3 a}$ & $\mathrm{CH}(\mathrm{COOMe})_{2}$ & $\mathrm{NaOAc}$ & $\mathrm{CH}_{2} \mathrm{Cl}_{2}$ & $\mathrm{rt}$ & 15 & $>99$ & 78 \\
\hline 4 & $(S)-\mathbf{3 a}$ & $\mathrm{CH}(\mathrm{COOMe})_{2}$ & $\mathrm{RbOAc}$ & $\mathrm{CH}_{2} \mathrm{Cl}_{2}$ & $\mathrm{rt}$ & 15 & $>99$ & 81 \\
\hline 5 & $(S)-\mathbf{3 a}$ & $\mathrm{CH}(\mathrm{COOMe})_{2}$ & $\mathrm{CsOAc}$ & $\mathrm{CH}_{2} \mathrm{Cl}_{2}$ & $\mathrm{rt}$ & 15 & $>99$ & 76 \\
\hline 6 & $(S)-\mathbf{3 a}$ & $\mathrm{CH}(\mathrm{COOMe})_{2}$ & $\mathrm{KOAC}$ & THF & $\mathrm{rt}$ & 15 & $>99$ & 55 \\
\hline 7 & $(S)-\mathbf{3 a}$ & $\mathrm{CH}(\mathrm{COOMe})_{2}$ & $\mathrm{KOAC}$ & toluene & $\mathrm{rt}$ & 15 & 52 & 61 \\
\hline 8 & $(S)-\mathbf{3 a}$ & $\mathrm{CH}(\mathrm{COOMe})_{2}$ & $\mathrm{KOAC}$ & $\left(\mathrm{ClCH}_{2}\right)_{2}$ & $\mathrm{rt}$ & 15 & $>99$ & 75 \\
\hline 9 & $(S)-\mathbf{3 a}$ & $\mathrm{CH}(\mathrm{COOMe})_{2}$ & KOAC & $\mathrm{CH}_{2} \mathrm{Cl}_{2}$ & $0^{\circ} \mathrm{C}$ & 96 & $>99$ & 61 \\
\hline 10 & $(R)-\mathbf{3 b}$ & $\mathrm{CH}(\mathrm{COOMe})_{2}$ & $\mathrm{KOAc}$ & $\mathrm{CH}_{2} \mathrm{Cl}_{2}$ & rt & 17 & $>99$ & 91 \\
\hline 11 & $(R)-\mathbf{3 b}$ & $\mathrm{CH}(\mathrm{COOMe})_{2}$ & $\mathrm{KOAC}$ & $\mathrm{CH}_{2} \mathrm{Cl}_{2}$ & $0^{\circ} \mathrm{C}$ & 105 & $>99$ & 96 \\
\hline 12 & $(S)-3 c$ & $\mathrm{CH}(\mathrm{COOMe})_{2}$ & $\mathrm{KOAC}$ & $\mathrm{CH}_{2} \mathrm{Cl}_{2}$ & rt & 17 & $>99$ & $r a c$ \\
\hline 13 & $(S)-\mathbf{3 a}$ & $\mathrm{CH}\left(\mathrm{COO}^{\prime} \mathrm{Bu}\right)_{2}$ & KOAC & $\mathrm{CH}_{2} \mathrm{Cl}_{2}$ & rt & 40 & 97 & 83 \\
\hline 14 & $(R)-\mathbf{3 b}$ & $\mathrm{CH}\left(\mathrm{COO}^{\prime} \mathrm{Bu}\right)_{2}$ & $\mathrm{KOAc}$ & $\mathrm{CH}_{2} \mathrm{Cl}_{2}$ & rt & 40 & 98 & 93 \\
\hline 15 & $(R)-\mathbf{3 b}$ & $\mathrm{CH}\left(\mathrm{COO}^{t} \mathrm{Bu}\right)_{2}$ & $\mathrm{KOAC}$ & $\mathrm{CH}_{2} \mathrm{Cl}_{2}$ & $0^{\circ} \mathrm{C}$ & 163 & 97 & 96 \\
\hline 16 & $(S)-\mathbf{3 a}$ & $\mathrm{C}(\mathrm{NHAc})(\mathrm{COOMe})_{2}$ & $\mathrm{KOAC}$ & $\mathrm{CH}_{2} \mathrm{Cl}_{2}$ & rt & 39 & 92 & 81 \\
\hline 17 & $(R)-\mathbf{3 b}$ & $\mathrm{C}(\mathrm{NHAc})(\mathrm{COOMe})_{2}$ & $\mathrm{KOAc}$ & $\mathrm{CH}_{2} \mathrm{Cl}_{2}$ & rt & 39 & 99 & 79 \\
\hline 18 & $(S)-\mathbf{3 a}$ & $\mathrm{CH}(\mathrm{COMe})_{2}$ & $\mathrm{KOAC}$ & $\mathrm{CH}_{2} \mathrm{Cl}_{2}$ & rt & 41 & 21 & 65 \\
\hline 19 & $(R)-3 \mathbf{b}$ & $\mathrm{CH}(\mathrm{COMe})_{2}$ & $\mathrm{KOAC}$ & $\mathrm{CH}_{2} \mathrm{Cl}_{2}$ & rt & 39 & 95 & 89 \\
\hline
\end{tabular}

${ }^{a}$ Yield of isolated product. ${ }^{b}$ Determined by HPLC analysis employing Daicel CHIRALCEL OD-H or CHIRALPAK AD.

(d) Torraca, K. E.; Kuwabe, S.; Buchwald, S. L. J. Am. Chem. Soc. 2000, 122, 12907. (e) Wolfe, J. P.; Tomori, H.; Sadighi, J. P.; Yin, J.; Buchwald, S. L. J. Org. Chem. 2000, 65, 1158. (f) Ueda, M.; Miyaura, N. J. Org. Chem. 2000, 65, 4450.

(5) (a) Imamoto, T.; Kusumoto, T.; Suzuki, N.; Sato, K. J. Am. Chem. Soc. 1985, 107, 5301. (b) Imamoto, T.; Oshiki, T.; Onozawa, T.; Kusumoto, T.; Sato, K. J. Am. Chem. Soc. 1990, 112, 5244. (c) Imamoto, T. Pure Appl. Chem. 1993, 65, 655.

(6) The mixtures of $\left(R_{\mathrm{P}}\right)-\mathbf{1 a}$ and $\left(S_{\mathrm{P}}\right)-\mathbf{1 a},\left(R_{\mathrm{P}}\right)-\mathbf{1 b}$ and $\left(S_{\mathrm{P}}\right)-\mathbf{1 b}$, and $\left(R_{\mathrm{P}}\right)-\mathbf{1 c}$ and $\left(S_{\mathrm{P}}\right)-\mathbf{1 c}$ were separated by preparative HPLC using ODS-column. Diasteromerically pure $\left(R_{\mathrm{P}}\right)-\mathbf{1 b},\left(S_{\mathrm{P}}\right)-\mathbf{1 b}$, $\left(R_{\mathrm{P}}\right)-\mathbf{1 c}$, and $\left(S_{\mathrm{P}}\right)$-1c were obtained also by fractional recrystallization.

$\left(R_{\mathrm{P}}\right)-1 \mathrm{a}$ : Colorless syrup; $[\alpha]_{\mathrm{D}}{ }^{25}-46.0^{\circ}\left(c 0.97, \mathrm{CHCl}_{3}\right) ;{ }^{1} \mathrm{H}$ NMR $\left(\mathrm{CDCl}_{3}\right) \delta 0.08-1.37(\mathrm{~m}, 8 \mathrm{H}), 0.77(\mathrm{~d}, J=4.6 \mathrm{~Hz}, 3 \mathrm{H})$, $0.79(\mathrm{~d}, J=4.8 \mathrm{~Hz}, 3 \mathrm{H}), 0.92(\mathrm{~d}, J=7.0 \mathrm{~Hz}, 3 \mathrm{H}), 0.98(\mathrm{~d}$, $J=14.3 \mathrm{~Hz}, 9 \mathrm{H}), 1.60-1.64(\mathrm{~m}, 2 \mathrm{H}), 1.96-1.99(\mathrm{~m}, 1 \mathrm{H})$, $2.21-2.24(\mathrm{~m}, 1 \mathrm{H}), 4.16-4.23(\mathrm{~m}, 1 \mathrm{H}), 7.20-7.48(\mathrm{~m}, 8 \mathrm{H})$, $7.95(\mathrm{ddd}, J=9.2,7.7,1.5 \mathrm{~Hz}, 1 \mathrm{H}) ;{ }^{13} \mathrm{C}$ NMR $\left(\mathrm{CDCl}_{3}\right) \delta 15.5$, 21.1, 22.2, 22.6, 25.1, 25.3, 31.4, 33.9, 34.3, 34.4, 43.3, 49.1, $49.2,79.3,125.7,125.8,126.8,127.4,129.8,130.2,130.4$, $130.9,132.2,132.3,133.4,141.1,146.9,147.0 ;{ }^{11} \mathrm{~B}\left(\mathrm{CDCl}_{3}\right) \delta$
-59.3 (brs); ${ }^{31} \mathrm{P}$ NMR $\left(\mathrm{CDCl}_{3}\right) \delta 114.3$ (m); IR (Neat) 3055, 2950, 2390, 1575, 1465, 1365, 1070, 985, 755, $700 \mathrm{~cm}^{-1}$; HRMS calced for $\mathrm{C}_{26} \mathrm{H}_{40}$ BKOP 449.2547 found 449.2514 . $\left(S_{\mathrm{P}}\right)-1 \mathrm{a}$ : Colorless syrup; $[\alpha]_{\mathrm{D}}{ }^{25}-59.3^{\circ}\left(c 0.91, \mathrm{CHCl}_{3}\right) ;{ }^{1} \mathrm{H}$ NMR $\left(\mathrm{CDCl}_{3}\right) \delta 0.07-1.43(\mathrm{~m}, 8 \mathrm{H}), 0.61(\mathrm{~d}, J=6.8 \mathrm{~Hz}, 3 \mathrm{H})$, $0.73(\mathrm{~d}, J=7.0 \mathrm{~Hz}, 3 \mathrm{H}), 0.73(\mathrm{~d}, J=6.5 \mathrm{~Hz}, 3 \mathrm{H}), 1.00(\mathrm{~d}$, $J=14.5 \mathrm{~Hz}, 9 \mathrm{H}), 1.57-1.64(\mathrm{~m}, 2 \mathrm{H}), 1.71-1.78(\mathrm{~m}, 1 \mathrm{H})$, $2.07-2.10(\mathrm{~m}, 1 \mathrm{H}), 4.18-4.23(\mathrm{~m}, 1 \mathrm{H}), 7.18-7.48(\mathrm{~m}, 8 \mathrm{H})$, 7.94 (ddd, $J=9.2,7.7,1.7 \mathrm{~Hz}, 1 \mathrm{H}) ;{ }^{13} \mathrm{C} \mathrm{NMR}\left(\mathrm{CDCl}_{3}\right) \delta 15.7$, 21.0, 22.2 22.7, 25.3, 25.6, 31.5, 33.9, 34.0, 34.3, 43.9, 48.9, 80.0, 125.5, 125.6, 126.8, 127.3, 128.9, 129.4, 130.3, 130.7, 132.0, 132.1, 133.5, 141.2, 147.1, 147.2; ${ }^{11} \mathrm{~B}$ NMR $\left(\mathrm{CDCl}_{3}\right) \delta$ -59.7 (brs); ${ }^{31} \mathrm{P}$ NMR $\left(\mathrm{CDCl}_{3}\right) \delta 120.4$ (m); IR (Neat) 3055, 2955, 2395, 1585, 1455, 1065, 950, 755, $\mathrm{cm}^{-1}$; HRMS calced for $\mathrm{C}_{26} \mathrm{H}_{40} \mathrm{BKOP} 449.2547$ found 449.2557 .

$\left(R_{\mathrm{P}}\right)$-1b: Colorless crystals; $\mathrm{mp} 186.5-187.5^{\circ} \mathrm{C} ;[\alpha]_{\mathrm{D}}{ }^{26}$ $-13.8^{\circ}\left(c 1.2, \mathrm{CHCl}_{3}\right) ;{ }^{1} \mathrm{H}$ NMR $\left(\mathrm{CDCl}_{3}\right) \delta 0.58-1.64(\mathrm{~m}$, $21 \mathrm{H}), 0.75(\mathrm{~d}, J=4.1 \mathrm{~Hz}, 3 \mathrm{H}), 0.76(\mathrm{~d}, J=4.1 \mathrm{~Hz}, 3 \mathrm{H}), 0.90$ $(\mathrm{d}, J=7.2 \mathrm{~Hz}, 3 \mathrm{H}), 1.98-2.06(\mathrm{~m}, 2 \mathrm{H}), 4.06-4.14(\mathrm{~m}, 1 \mathrm{H})$, $7.26-7.54(\mathrm{~m}, 8 \mathrm{H}), 8.18(\mathrm{ddd}, J=12.8,7.7,1.2 \mathrm{~Hz}, 1 \mathrm{H}) ;{ }^{13} \mathrm{C}$ $\operatorname{NMR}\left(\mathrm{CDCl}_{3}\right) \delta 15.6,21.0,22.0,22.9,25.2,25.3,25.3,25.7$, 25.7, 26.0, 26.2 26.5, 26.6, 31.3, 34.3, 36.1, 36.6, 42.8, 43.3, 
$49.4,49.5,79.3,79.4,127.0,127.2,127.2,127.7,130.2$, 130.4, 130.9, 131.2, 131.3, 131.4, 135.2, 135.4, 141.0, 141.0, 146.0; ${ }^{11} \mathrm{~B}$ NMR $\left(\mathrm{CDCl}_{3}\right) \delta-61.8(\mathrm{~d}, J=64.5 \mathrm{~Hz}) ;{ }^{31} \mathrm{P} \mathrm{NMR}$ $\left(\mathrm{CDCl}_{3}\right) \delta 122.7(\mathrm{~m})$; IR (KBr) 3050, 2935, 2395, 1590, 1560, 985, 775, 705, $\mathrm{cm}^{-1}$; FAB MS m/z $433\left(\mathrm{M}^{+}-3 \mathrm{H}\right)$; Anal. Calcd for $\mathrm{C}_{28} \mathrm{H}_{42} \mathrm{BOP}$ : C, 77.06; H, 9.70. Found: C, $77.14 \mathrm{H}, 9.79$. $\left(S_{\mathrm{P}}\right)$-1b: Colorless crystals; mp $148.0-149.0^{\circ} \mathrm{C} ;[\alpha]_{\mathrm{D}}{ }^{25}-$ 93.7 $\left(c 1.04, \mathrm{CHCl}_{3}\right) ;{ }^{1} \mathrm{H} \mathrm{NMR}\left(\mathrm{CDCl}_{3}\right) \delta 0.32-1.67(\mathrm{~m}$, $22 \mathrm{H}), 0.49(\mathrm{~d}, J=6.8 \mathrm{~Hz}, 3 \mathrm{H}), 0.70(\mathrm{~d}, J=7.0 \mathrm{~Hz}, 3 \mathrm{H}), 0.91$ $(\mathrm{d}, J=6.5 \mathrm{~Hz}, 3 \mathrm{H}), 2.03-2.06(\mathrm{~m}, 1 \mathrm{H}), 4.12-4.21(\mathrm{~m}, 1 \mathrm{H})$, $7.26-7.54(\mathrm{~m}, 8 \mathrm{H}), 8.22(\mathrm{dd}, J=14.3,7.7 \mathrm{~Hz}, 1 \mathrm{H}) ;{ }^{13} \mathrm{C} \mathrm{NMR}$ $\left(\mathrm{CDCl}_{3}\right) \delta 15.5,21.0,22.1,22.5,25.5,25.6,25.7,25.9,26.1$, 26.5, 26.7, 31.4, 34.0, 35.3, 35.8, 43.6, 48.8, 48.8, 79.5, 79.6, 126.9, 127.0, 127.6, 127.7, 129.0, 129.5, 129.8, 131.4, 131.4, $135.7,135.9,141.4,141.5,145.5 ;{ }^{11} \mathrm{~B}\left(\mathrm{CDCl}_{3}\right) \delta-62.1(\mathrm{~d}$, $J=66.8 \mathrm{~Hz}) ;{ }^{31} \mathrm{P}$ NMR $\left(\mathrm{CDCl}_{3}\right) \delta 117.2(\mathrm{~m}) ; \mathrm{IR}(\mathrm{KBr}) 3055$, 2930, 2385, 1560, 1445, 980, 755, $695 \mathrm{~cm}^{-1}$; FAB MS m/z $433\left(\mathrm{M}^{+}-3 \mathrm{H}\right)$; Anal. Calcd for $\mathrm{C}_{28} \mathrm{H}_{42} \mathrm{BOP}$ : C, 77.06; H, 9.70. Found: C, 77.27; H, 9.72.

$\left(R_{\mathrm{P}}\right)$-1c: Colorless crystals; $\mathrm{mp} 130.5-131.0{ }^{\circ} \mathrm{C} ;[\alpha]_{\mathrm{D}}{ }^{26}$ $-23.5^{\circ}\left(c 0.82, \mathrm{CHCl}_{3}\right) ;{ }^{1} \mathrm{H} \mathrm{NMR}\left(\mathrm{CDCl}_{3}\right) \delta 0.34-1.64(\mathrm{~m}$, $10 \mathrm{H}), 0.51(\mathrm{~d}, J=6.8 \mathrm{~Hz}, 3 \mathrm{H}), 0.81(\mathrm{~d}, J=7.0 \mathrm{~Hz}, 3 \mathrm{H}), 0.85$ $(\mathrm{d}, J=6.5 \mathrm{~Hz}, 3 \mathrm{H}), 1.81-1.89(\mathrm{~m}, 1 \mathrm{H}), 2.16-2.19(\mathrm{~m}, 1 \mathrm{H})$, $4.19-4.27(\mathrm{~m}, 1 \mathrm{H}), 6.82-6.84(\mathrm{~m}, 2 \mathrm{H}), 7.00-7.04(\mathrm{~m}, 2 \mathrm{H})$, 7.13-7.30 (m, 7H), 7.48-7.55 (m, 2H), 8.23-8.28 (m, 1H); ${ }^{13} \mathrm{C} \mathrm{NMR}\left(\mathrm{CDCl}_{3}\right) \delta 15.2,21.0,21.0,22.1,22.6,25.3,25.4$, 31.4, 34.2, 43.3, 49.0, 49.0, 79.9, 126.8, 126.9, 127.0, 127.7, $127.8,129.9,130.3,130.4,131.3,131.6,131.7,132.1,132.7$, 133.5, 133.6, 133.7, 133.7, 134.3, 140.4, 140.4, 146.5, 146.5; ${ }^{11} \mathrm{~B} \mathrm{NMR}\left(\mathrm{CDCl}_{3}\right) \delta-57.5(\mathrm{~d}, J=55.7 \mathrm{~Hz}) ;{ }^{31} \mathrm{P} \mathrm{NMR}\left(\mathrm{CDCl}_{3}\right)$ $\delta 101.3(\mathrm{~m})$; IR (KBr) 3055, 2945, 2420, 1590, 1440, 1060, 975, 775, 755, $700 \mathrm{~cm}^{-1}$; FAB MS m/z $427\left(\mathrm{M}^{+}-3 \mathrm{H}\right)$; Anal. Calcd for $\mathrm{C}_{28} \mathrm{H}_{36}$ BOP: C, 78.14; $\mathrm{H}, 8.43$. Found: $\mathrm{C}, 78.13 \mathrm{H}$, 8.49

$\left(S_{\mathrm{P}}\right)-1 \mathrm{c}$ : Colorless crystals; $\mathrm{mp} 105.5-106.5^{\circ} \mathrm{C} ;[\alpha]_{\mathrm{D}}{ }^{25}-84.7^{\circ}$ (c $\left.0.84, \mathrm{CHCl}_{3}\right) ;{ }^{1} \mathrm{H}$ NMR $\left(\mathrm{CDCl}_{3}\right) \delta 0.50-1.69(\mathrm{~m}, 10 \mathrm{H})$, $0.73(\mathrm{~d}, J=6.8 \mathrm{~Hz}, 3 \mathrm{H}), 0.82(\mathrm{~d}, J=6.5 \mathrm{~Hz}, 3 \mathrm{H}), 0.85(\mathrm{~d}$, $J=7.0 \mathrm{~Hz}, 3 \mathrm{H}), 1.84-1.92(\mathrm{~m}, 2 \mathrm{H}), 4.34-4.42(\mathrm{~m}, 1 \mathrm{H})$, 6.74-6.76 (m, 2H), 6.97-7.24 (m, 9H), 7.48-7.55 (m, 2H), $8.32-8.38(\mathrm{~m}, 1 \mathrm{H}) ;{ }^{13} \mathrm{C} \mathrm{NMR}\left(\mathrm{CDCl}_{3}\right) \delta 15.6,21.2,22.1$, 22.7, 25.8, 31.4, 34.1, 43.7, 48.9, 49.0, 80.8, 80.8, 126.8, 126.9, 126.9, 127.6, 127.7, 129.8, 129.8, 130.0, 130.0, 130.0, 131.3, 131.6, 131.6, 131.7, 132.0, 133.9, 134.1, 134.2, 134.6, $134.9,140.4,140.5,146.5,146.5 ;{ }^{11} \mathrm{~B}\left(\mathrm{CDCl}_{3}\right) \delta-60.7(\mathrm{~d}$, $J=63.4 \mathrm{~Hz}) ;{ }^{31} \mathrm{P} \mathrm{NMR}\left(\mathrm{CDCl}_{3}\right) \delta 104.2(\mathrm{~m}) ; \mathrm{IR}(\mathrm{KBr}) 3065$, 2955, 2375, 1435, 1130, 1060, 1010, 755, $695 \mathrm{~cm}^{-1}$; FAB MS $\mathrm{m} / \mathrm{z} 427\left(\mathrm{M}^{+}-3 \mathrm{H}\right)$; Anal. Calcd for $\mathrm{C}_{28} \mathrm{H}_{42} \mathrm{BOP}$ : C, 78.14; $\mathrm{H}$, 8.43. Found: C, 78.26; $\mathrm{H}, 8.48$.

(7) (S)-2a: Colorless thin plate; $\mathrm{mp} 112.5-113.5^{\circ} \mathrm{C} ;[\alpha]_{\mathrm{D}}{ }^{25}$ $-11.4^{\circ}\left(c 0.68, \mathrm{CHCl}_{3}\right) ;{ }^{1} \mathrm{H} \mathrm{NMR}\left(\mathrm{CDCl}_{3}\right) \delta 0.38-1.26(\mathrm{~m}$, $3 \mathrm{H}), 0.89(\mathrm{~d}, J=10.1 \mathrm{~Hz}, 3 \mathrm{H}), 1.05(\mathrm{~d}, J=13.8 \mathrm{~Hz}, 9 \mathrm{H})$, 7.15-7.65 (m, 8H), 8.01-8.09 (m, 1H); ${ }^{13} \mathrm{C} \mathrm{NMR}\left(\mathrm{CDCl}_{3}\right) \delta$ $7.5,7.5,7.8,7.8,25.8,25.8,25.9,29.7,30.0,125.0,125.4$,
126.6, 126.8, 127.4, 127.7, 129.3, 130.0, 130.6, 131.7, 131.8, 136.0,

136.1, 136.2, 136.2, 141.6, 141.6, 146.4; ${ }^{11} \mathrm{~B}$ NMR $\left(\mathrm{CDCl}_{3}\right) \delta$ $-56.8(\mathrm{~d}, J=60.9 \mathrm{~Hz}) ;{ }^{31} \mathrm{P} \mathrm{NMR}\left(\mathrm{CDCl}_{3}\right) \delta 31.9(\mathrm{~m}) ; \mathrm{IR}$ (KBr) 3050, 2970, 2380, 1555, 1465, 1075, 1070, 765, 710 $\mathrm{cm}^{-1}$; FAB MS m/z $267\left(\mathrm{M}^{+}-3 \mathrm{H}\right)$; Anal. Calcd for $\mathrm{C}_{17} \mathrm{H}_{24} \mathrm{BP}$ : C, 75.58; H, 8.95. Found: C, 75.60; H, 8.94.

$(R)-2 b$ : Colorless prism; mp $131.0-132.0^{\circ} \mathrm{C} ;[\alpha]_{\mathrm{D}}{ }^{26}-68.6^{\circ}(c$ $\left.0.84, \mathrm{CHCl}_{3}\right) ;{ }^{1} \mathrm{H}$ NMR $\left(\mathrm{CDCl}_{3}\right) \delta 0.34-1.73(\mathrm{~m}, 14 \mathrm{H}), 1.13$ $(\mathrm{d}, J=11.3 \mathrm{~Hz}, 3 \mathrm{H}), 7.20-7.30(\mathrm{~m}, 3 \mathrm{H}), 7.35-7.64(\mathrm{~m}, 5 \mathrm{H})$, 8.04 (ddd, $J=11.8,7.5,1.5 \mathrm{~Hz}, 1 \mathrm{H}) ;{ }^{13} \mathrm{C} \mathrm{NMR}\left(\mathrm{CDCl}_{3}\right) \delta 9.6$, 9.6, 10.0, 10.0, 25.6, 26.4, 26.5, 26.6, 34.3, 34.7, 126.5, 127.0, 127.3, 127.4, 127.8, 128.0, 129.4, 130.4, 130.4, 131.2, 131.3, 135.6, 135.8, 141.3, 141.4, 146.3; ${ }^{11} \mathrm{~B}$ NMR $\left(\mathrm{CDCl}_{3}\right) \delta-58.1$ $(\mathrm{d}, J=57.2 \mathrm{~Hz}) ;{ }^{31} \mathrm{P} \mathrm{NMR}\left(\mathrm{CDCl}_{3}\right) \delta 19.8(\mathrm{~m})$; IR $(\mathrm{KBr}) 3055$, 2935, 2360, 1465, 1445, 1065, 910, 765, $710 \mathrm{~cm}^{-1}$; FAB MS $\mathrm{m} / \mathrm{z} 293\left(\mathrm{M}^{+}-3 \mathrm{H}\right)$; Anal. Calcd for $\mathrm{C}_{19} \mathrm{H}_{26} \mathrm{BP}: \mathrm{C}, 77.05 ; \mathrm{H}$, 8.85. Found: C, 77.07; H, 8.82.

$(S)$-2c: Colorless prism; $\mathrm{mp} 124.0-124.5^{\circ} \mathrm{C} ;[\alpha]_{\mathrm{D}}{ }^{25} 54.8^{\circ}(c$ $\left.0.82, \mathrm{CHCl}_{3}\right) ;{ }^{1} \mathrm{H}$ NMR $\left(\mathrm{CDCl}_{3}\right) \delta 0.64-1.47(\mathrm{~m}, 3 \mathrm{H}), 1.43(\mathrm{~d}$, $J=10.1 \mathrm{~Hz}, 3 \mathrm{H}), 6.89-6.91(\mathrm{~m}, 2 \mathrm{H}), 7.11-7.70(\mathrm{~m}, 11 \mathrm{H})$, 7.99 (ddd, $J=13.5,7.7,1.7 \mathrm{~Hz}, 1 \mathrm{H}) ;{ }^{13} \mathrm{C}$ NMR $\left(\mathrm{CDCl}_{3}\right) \delta$ 11.0, 11.6, 11.7, 12.0, 127.2, 127.4, 127.5, 128.2, 128.3, $128.4,128.7,129.4,130.4,130.9,130.1,131.2,131.5,131.5$, 132.0, 132.6, 134.2, 134.2, 134.3, 134.4, 140.5, 140.5, 146.8, $146.9 ;{ }^{11} \mathrm{~B}$ NMR $\left(\mathrm{CDCl}_{3}\right) \delta-54.4(\mathrm{~d}, J=54.3 \mathrm{~Hz}) ;{ }^{31} \mathrm{P}$ NMR $\left(\mathrm{CDCl}_{3}\right) \delta 12.7$ (m); IR (KBr) 3055, 2935, 2360, 1465, 1445, 1065, 910, 765, $710 \mathrm{~cm}^{-1}$; FAB MS m/z $287\left(\mathrm{M}^{+}-3 \mathrm{H}\right)$; Anal. Calcd for $\mathrm{C}_{19} \mathrm{H}_{20} \mathrm{BP}$ : C, 78.65; H, 6.95. Found: C, 78.62; H, 6.88.

(8) Absolute configuration of compound $(R)-\mathbf{2 a}$, which was synthesized from $\left(R_{\mathrm{P}}\right)$-1a, was determined single crystal X-ray analysis using Flack parameter.

(9) Imamoto, T.; Kikuchi, S.; Miura, T.; Wada, Y. Org. Lett. 2001, 3, 87.

(10) 2-Biphenyl(t-butyl)menthyloxyphosphine-borane and 2-biphenyl(cyclohexyl)menthyloxyphosphine-borane were also allowed to react with methylithium. However, the former one did not react at $40{ }^{\circ} \mathrm{C}$ and the reaction of the latter one at reflux afforded many products.

(11) (a) Frost, C. G.; Howarth, J.; Williams, J. M. J. Tetrahedron: Asymmetry 1992, 3, 1089. (b) Trost, B. M.; van Vranken, D. L. Chem. Rev. 1996, 96, 395.

(12) For recent examples, (a) Nelson, S. G.; Hilfiker, M. A. Org. Lett. 1999, 1, 1379. (b) Graf, C.-D.; Malan, C.; Harms, K.; Knochel, P. J. Org. Chem. 1999, 64, 5581. (c) Fuji, K.; Ohnishi, H.; Moriyama, S.; Tanaka, K.; Kawabata, T.; Tsubaki, K. Synlett 2000, 351.

Article Identifier:

1437-2096,E;2001,0,SI,0999,1002,ftx,en;Y03901ST.pdf 